\title{
A MONOTONE PATH IN AN EDGE-ORDERED GRAPH
}

\author{
A. BIALOSTOCKI \\ Department of Mathematics and Applied Statistics \\ University of Idaho \\ Moscow, Idaho 83843 \\ and \\ Y. RODITTY \\ School of Mathematical Sciences \\ Tel-Aviv University \\ Te1-Aviv, Israel 69978 \\ (Received January 8, 1986 and in revised form September 23, 1986)
}

ABSTRACT. An edge-ordered graph is an ordered pair (G,f), where $G$ is a graph and $f$ is a bijective function, $f: E(G) \rightarrow\{1,2, \ldots,|E(G)|\}$. A monotone path of length $k$ in $(G, f)$ is a simple path $P_{k+1}: v_{1} v_{2} \ldots v_{k+1}$ in $G$ such that either $f\left(\left\{v_{i}, v_{i+1}\right\}\right)<f\left(\left\{v_{i+1}, v_{1+2}\right\}\right)$ or $f\left(\left\{v_{i}, v_{i+1}\right\}\right)>f\left(\left\{v_{i+1}, v_{i}\right\}\right)$ for $i=1,2, \ldots, k-1$.

It is proved that a graph $G$ has the property that $(G, f)$ contains a monotone path of length three for every $f$ iff $G$ contains as a subgraph, an odd cycle of length at least five or one of six listed graphs.

KEY WORDS AND PHRASES. Edge-ordered graph, monotone path.

1980 AMS SUBJECT CLASSIFICATION CODE. 05C55, 05C38.

1. INTRODUCTION.

Graphs in this paper are finite, loopless and have no multiple edges. We denote by $G=G(V, E)$ a graph with $E(G)$ as its edge-set of cardinality e(G) and $V(G)$ as its vertex-set. Let $K_{n}, P_{n}, C_{n}$ be the complete graph, the path and the cycle, on $n$ vertices, respectively. The vertex-chromatic number of $G$ is denoted by $X(G)$, and $d(v)$ is the degree of a vertex $v \in V(G)$. By $H \subset G$ we mean that $H$ is a subgraph of $G$ and $H \notin G$ is the negation of this fact.

Definitions and Notation

1. An edge-ordered graph is an ordered pair $(G, f)$, where $G$ is a graph and $f$ is a bijective function, $f: E(G) \rightarrow\{1,2,3, \ldots, e(G)\}$.

2. A monotone path of length $k, k \geqq 3$ in $(G, f)$, denoted by $\operatorname{MP}_{k+1}$, is a simple path $\mathrm{P}_{k+1}: \mathrm{v}_{1} \mathrm{v}_{2} \cdots \mathrm{v}_{\mathrm{k}+1}$ in $\mathrm{G}$ such that either

$$
f\left(\left\{v_{i}, v_{i+1}\right\}\right)<f\left(\left\{v_{i+1}, v_{i+2}\right\}\right)
$$


or

$$
f\left(\left\{v_{i}, v_{i+1}\right\}\right)>f\left(\left\{v_{i+1}, v_{i+2}\right\}\right) \text { for } i=1,2, \ldots, k-1 .
$$

3. We denote by $G \rightarrow M_{k}$ the fact that $(G, f)$ contains an $M_{k}$ for every function $f$, and let

$$
A_{k}=\left\{G \mid G \rightarrow M P_{k}\right\}, \quad k \geqq 3
$$

The following Theorem 1.1 is well known, see [1], [2], [3], for a proof and generalizations:

THEOREM 1.1. For every positive integer $k$, there is a minimal integer $g(k)$, such that $k_{n} \in A_{k}$ for every $n \geqq g(k)$.

The main result of this paper is:

THEOREM 1.2. A graph $G$ belongs to $A_{4}$ iff $G$ contains either $C_{2 n+1}, n \geqq 2$, or one of the following graphs:
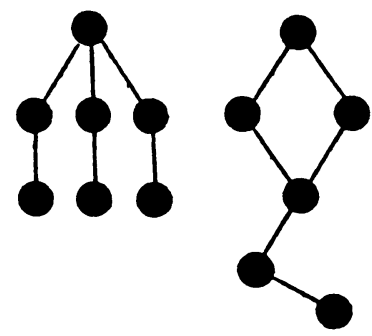

$\mathrm{H}_{2}$

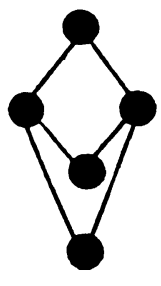

$H_{3}$

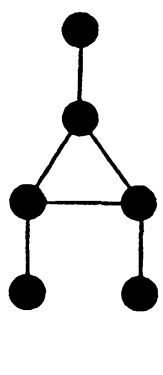

$\mathrm{H}_{4}$
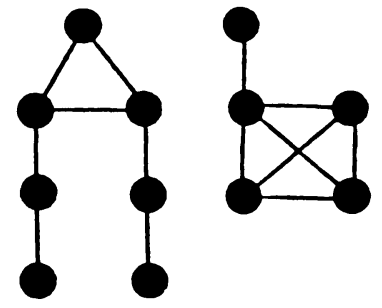

$H_{5}$

$H_{6}$

Fig. 1

REMARK. Notice that a graph $G$ belongs to $A_{3}$ iff $G$ contains a path $P_{3}$.

2. PROOFS

The following lemmas are essential for the proof of Theorem 1.2.

LEMMA 2.1. The graphs $\mathrm{H}_{1}, \mathrm{H}_{2}, \mathrm{H}_{3}, \mathrm{H}_{4}, \mathrm{H}_{5}, \mathrm{H}_{6}$, and $\mathrm{C}_{2 \mathrm{n}+1}$ where $\mathrm{n} \geq 2$ belong to $\mathrm{A}_{4}$.

PROOF. The proof is a straightforward verification for each of the graphs. We prove that $\mathrm{H}_{4} \in \mathrm{A}_{4}$. The proof of the remaining cases is similar. Assume that there is an $\mathrm{f}$ such that no $\mathrm{MP}_{4}$ occurs in $\left(\mathrm{H}_{4}, \mathrm{f}\right)$. It turns out that up to isomorphism, the integers $1,2,3$ can be assigned to the edges of $\mathrm{H}_{4}$ in the following 5 ways:
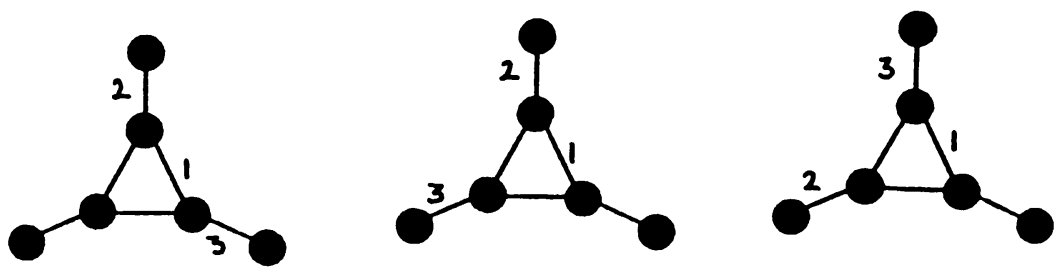

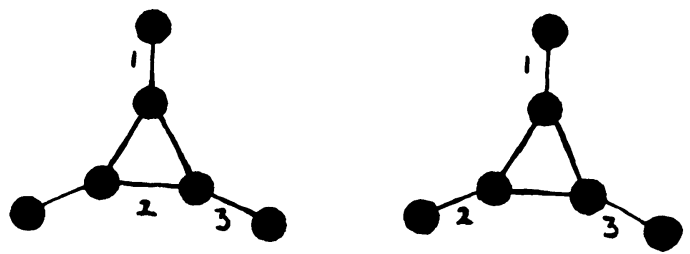

Now, one can see that in each case it is impossible to complete the labeling of the edges such that $\left(\mathrm{H}_{4}, \mathrm{f}\right)$ does not contain an $\mathrm{MP}_{4} \cdot$

The following definition is needed for the next lemma.

DEFINITION. Let $a, b, c_{1}, c_{2}, \ldots, c_{m+1}, a_{1}, \ldots, a_{2 n}$ be non-negative integers where $m \geqq 0$ and $n \geqq 2$. The graph $L_{1}\left(m, a, b, c c_{1}, c_{2}, \ldots, c_{m+1}\right), L_{2}(a, b), L_{3}(a, b)$, and $\mathrm{R}_{2 \mathrm{n}}\left(\mathrm{a}_{1}, \mathrm{a}_{2}, \ldots \mathrm{a}_{2 \mathrm{n}}\right)$ are defined in Fig. 2 .

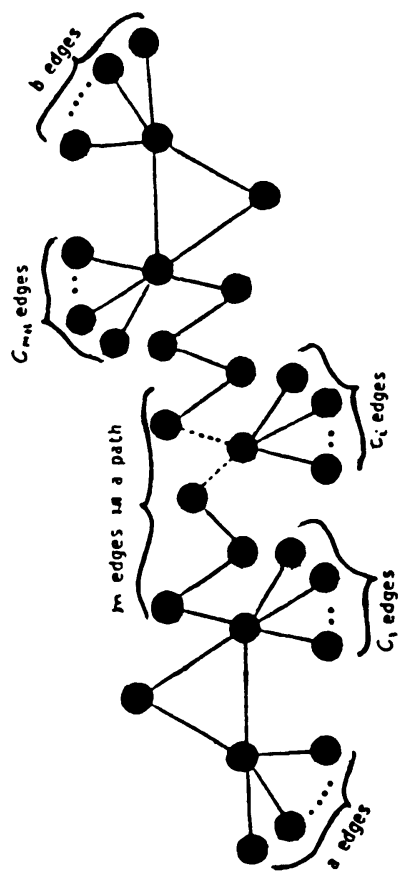

$L_{1}=L_{1}\left(m, a, b, c_{1}, c_{2}, \ldots, c_{m+1}\right)$

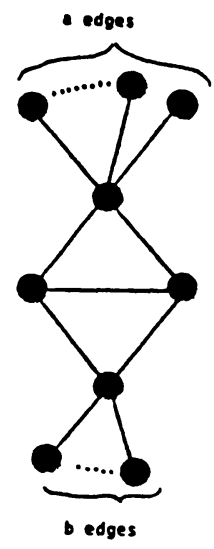

$t_{2}(a, b)$

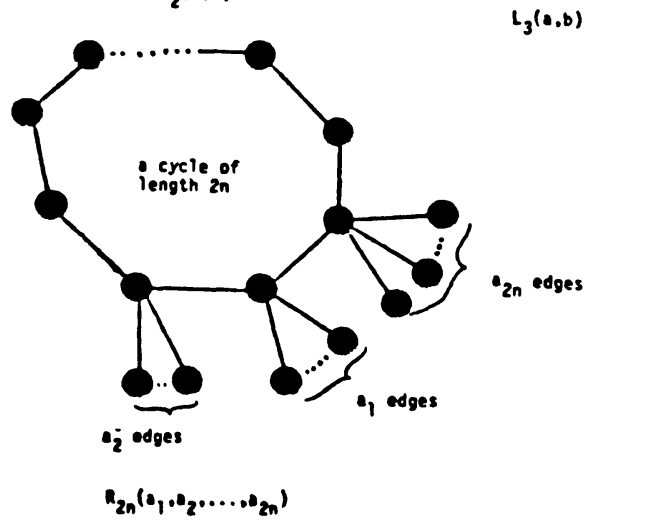

$\underline{F 1_{B} \cdot 2}$ 
LEMMA 2.2. (i). For all non-negative integers $a, b, c_{1}, c_{2}, \ldots, c_{m+1}, a_{1}, \ldots, a_{2 n}$ where $m \geqq 0$ and $n \geqq 2$, the graphs $L_{1}, L_{2}(a, b), L_{3}(a, b)$, and $R_{2 n}\left(a_{1}, a_{2}, \ldots, a_{2 n}\right)$ do not belong to $\mathrm{A}_{4}$.

(ii). The complete graph $\mathrm{K}_{4}$ does not belong to $\mathrm{A}_{4}$.

PROOF. We set $e$ for $e(G)$. For the proof of (1), a partial labeling of the edges of the graphs in question is presented in Fig. 3. The labeling of the remaining edges is arbitrary. An $\mathrm{MP}_{4}$ will not occur. A labeling of $E\left(K_{4}\right)$ is also presented in Fig. 3 .
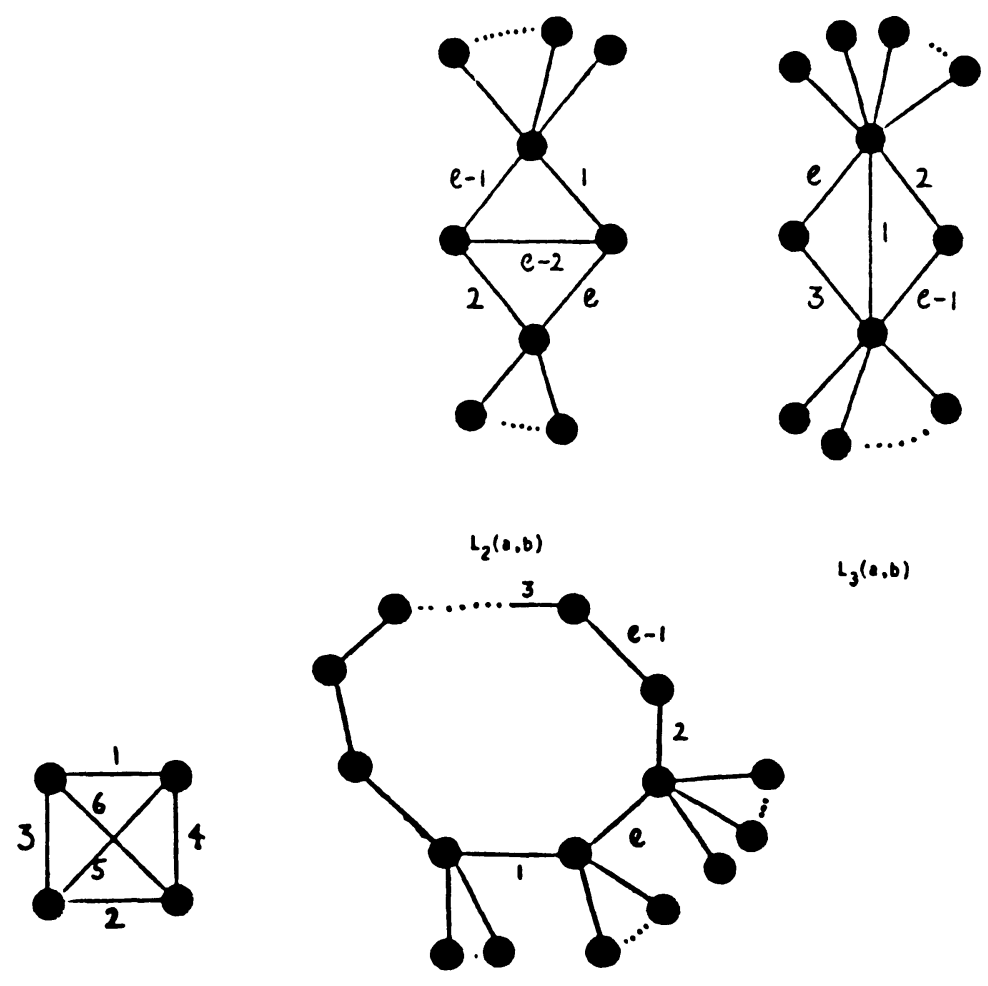

$L_{3}(a, b)$

$n_{2 n}\left(0,1 \cdot 0_{2} \ldots ., 0_{2 n}\right)$

Fig. $3 a$ 


$$
\begin{aligned}
& x= \begin{cases}e-1-\frac{m}{2}, & m \text { even } \\
\frac{m+1}{2}+2, & m \text { odd }\end{cases} \\
& y= \begin{cases}e-2-\frac{m}{2}, & m \text { even } \\
\frac{c+1}{2}+3, & m \text { odd }\end{cases} \\
& z= \begin{cases}\frac{m+2}{2}, & m \text { odd } \\
e-1-\frac{m+1}{2} & ,\end{cases}
\end{aligned}
$$
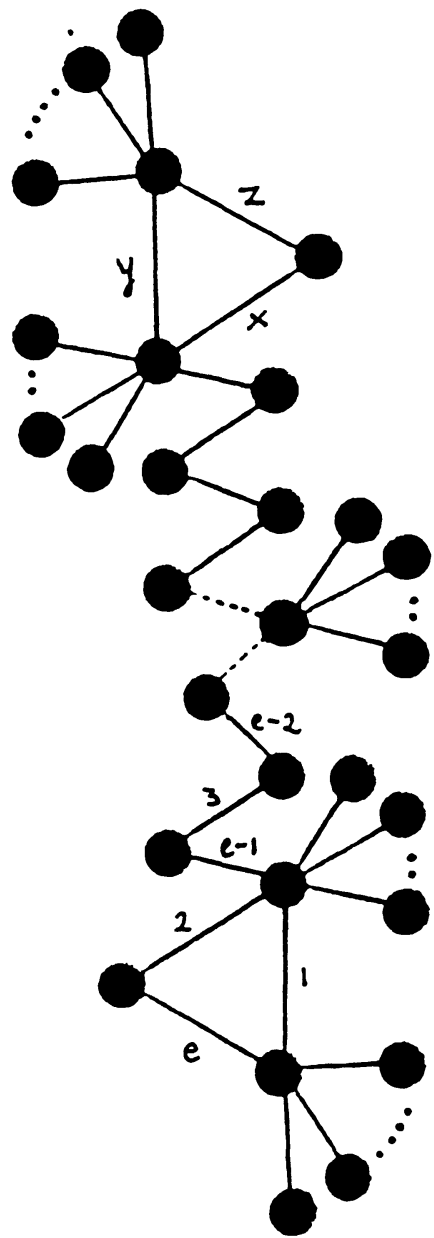

$$
L_{1}=L_{1}\left(m, a, b, c_{1}, c_{2}, \ldots, c_{m+1}\right)
$$

Fig. 3 b

PROOF OF THEOREM 1.2. Clearly, every graph $G$ that contains $C_{2 n+1}$, $n \geqq 2$, or an $H_{i}, i=1, \ldots, 6$ belongs to $A_{4}$. To prove the opposite containment let $G \in A_{4}$. We may assume that $G$ is connected and contains a $P_{4}$, hence $\chi(G) \geqq 2$. We cousider two cases: $X(G)=2$ and $\chi(G) \geqq 3$.

CASE 1. Let $X(G)=2$. If $G$ is a tree, let $P_{t}: x_{1} x_{2} \ldots \ldots x_{t}$ be its longest path. If $t=4$, then $G$ is double star yielding $G \notin A_{4}$, a contradiction. Hence, $t \geqq 5$. Note that the maximality of $P_{t}$ implies that there is no vertex-disjoint path to $P_{t}$, say $P_{n}$, where $n \geqq 3$, with initial vertex $x_{2}$ or $x_{t-1}$. If for a certain $i$, $3 \leqq i \leqq t-2$ there is a vertex-disjoint path to $P_{t}$, say $P_{m}$, where $m \geqq 3$, whose initial vertex is $x_{1}$, then $H_{1} \subset G$, and we are through. Otherwise, $G$ can be embedded 
in a graph $\mathrm{R}_{2 \mathrm{n}}\left(\mathrm{a}_{1}, \mathrm{a}_{2}, \ldots, \mathrm{a}_{2 \mathrm{n}}\right)$ for a certain $\mathrm{n}$ and non-negative integers $a_{1}, a_{2}, \ldots, a_{2 n}$ and in view of Lemma $2.2, G \notin A_{4}, a$ contradiction. Thus we may assume that $G$ is not a tree.

Let $\mathrm{C}_{2 \mathrm{t}}$ be the shortest cycle in $\mathrm{G}$. Assume first $t=2$, i.e., $\mathrm{C}_{2 t}$ is a 4-cycle. One can see that if $\mathrm{H}_{2} \notin \mathrm{G}$ and $\mathrm{H}_{3} \notin \mathrm{G}$ then $\mathrm{G}=\mathrm{R}_{4}\left(\mathrm{a}_{1}, \mathrm{a}_{2}, \mathrm{a}_{3}, \mathrm{a}_{4}\right)$ for some non-negative integers $a_{1}, a_{2}, a_{3}, a_{4}$ and hence by Lemma $2.2, G \notin A_{4}$, a contradiction. Thus we may assume that $t \geqq 3$. Similarly in view of the minimality of $C_{2 t}$ it follows that if $H_{1} \notin G$ then $G=R_{2 t}\left(a_{1}, a_{2}, \ldots, a_{2 t}\right)$ for some non-negative integers $a_{1}, a_{2}, \ldots, a_{2 t}$ implying that $G \notin A_{4}$, a contradiction. Hence, the proof of Case 1 is completed.

CASE 2. Let $\chi(G) \geqq 3$. Hence $G$ contains an odd cycle $C_{2 n+1}$. If $n \geq 2$ then we are through. So we may assume that $G$ contains only triangles. Let $C_{3}$ be any triangle in $G$ with a vertex-set $\{x, y, z\}$. Consider two cases:

(i) Let $d(x), d(y), d(z) \geqq 3$. It follows that either $H_{4} \subset G$ and we are through, or $\mathrm{K}_{4} \subset \mathrm{G}$ or $\mathrm{L}_{2}(0,1) \subset \mathrm{G}$. By Lemma $2.2, \mathrm{G} \neq \mathrm{K}_{4}$, hence $\mathrm{K}_{4} \subset \mathrm{G}$ implies that $\mathrm{H}_{6} \subset \mathrm{G}$. Again Lemma 2.2, $\mathrm{G} \neq \mathrm{L}_{2}(\mathrm{a}, \mathrm{b})$ for a11 non-negative integers $a$ and $b$. Hence $\mathrm{L}_{2}(0,1) \subset G$ implies that one of the graphs $\mathrm{H}_{2}, \mathrm{H}_{4}$, or $\mathrm{H}_{6}$ is contained in $G$. This completes the proof of case (i).

(ii) Assume that at least one of the vertices $x, y, z$ is of degree 2. By Lemma 2.2, $G$ is not a subgraph of $L_{1}$ or $L_{2}(0, b)$ or $L_{3}(a, b)$ for any non-negative integers $a, b$, and $c$; hence $G$ must contain one of the graphs $\mathrm{H}_{1}, \mathrm{H}_{2}, \mathrm{H}_{3}$, or $\mathrm{H}_{5}$. This completes the proof of case (ii) and of the theorem.

\section{REFERENCES}

1. BIALOSTOCKI, A. An Analog of the Erdös-Szekeres Theorem. Submitted.

2. CALDERBANK, A.R., CHUNG, F.R.K. and STURTEVANT, D.G. Increasing Sequences with Nonzero Block Sums and Increasing Paths in Edge-Ordered Graphs, Discrete Math $\underline{50}(1984), 15-28$.

3. CHVÁtAL, V. and KOMLÓS, J. Some Combinatorial Theorems on Monotonicity, Canad. Math. Bul1. 14 (1971), 151-157. 


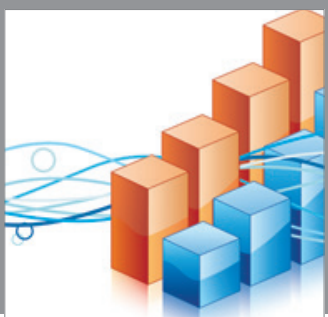

Advances in

Operations Research

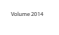

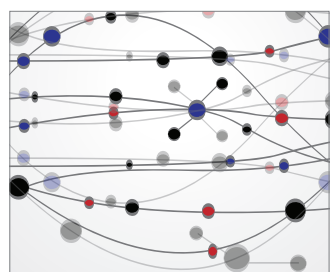

\section{The Scientific} World Journal
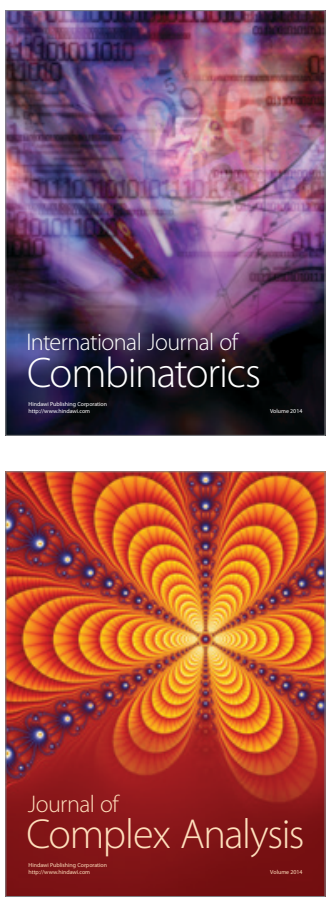

International Journal of

Mathematics and

Mathematical

Sciences
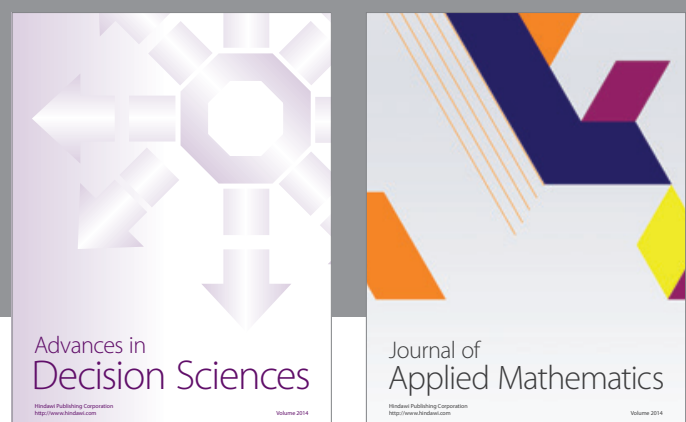

Journal of

Applied Mathematics
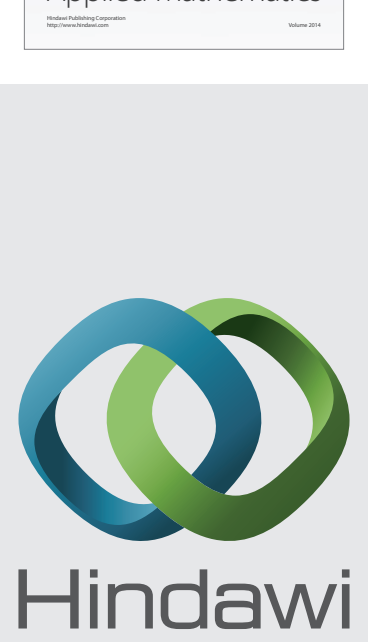

Submit your manuscripts at http://www.hindawi.com
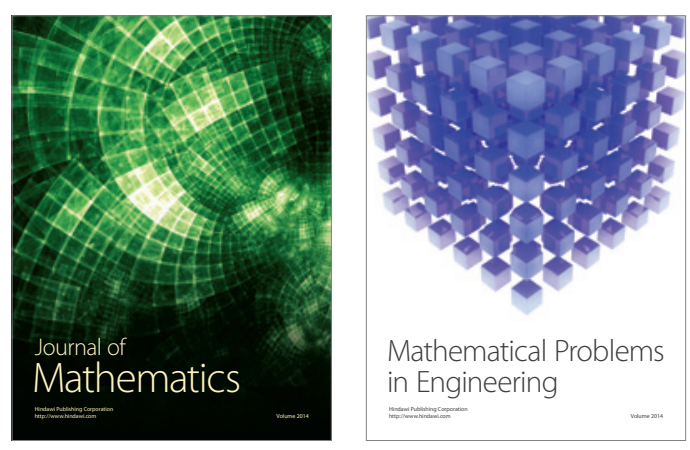

Mathematical Problems in Engineering
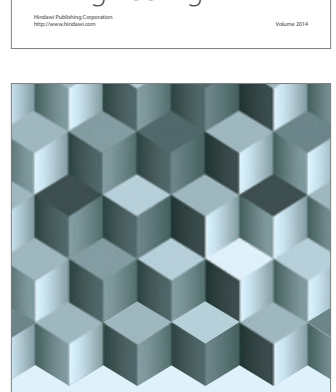

Journal of

Function Spaces
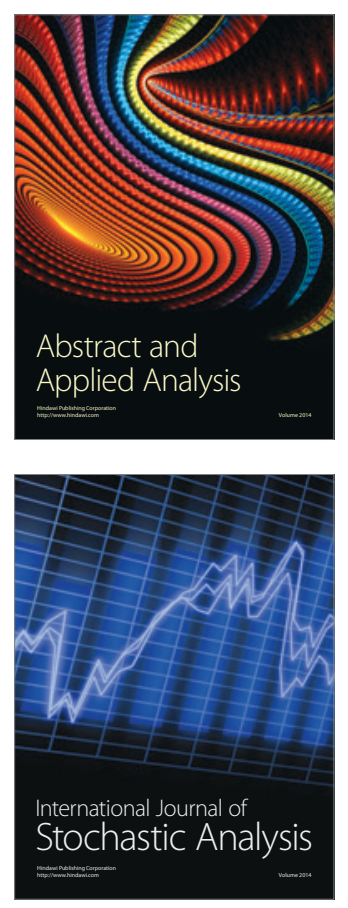

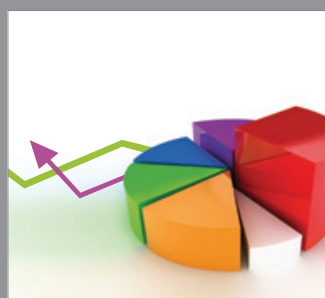

ournal of

Probability and Statistics

Promensencen
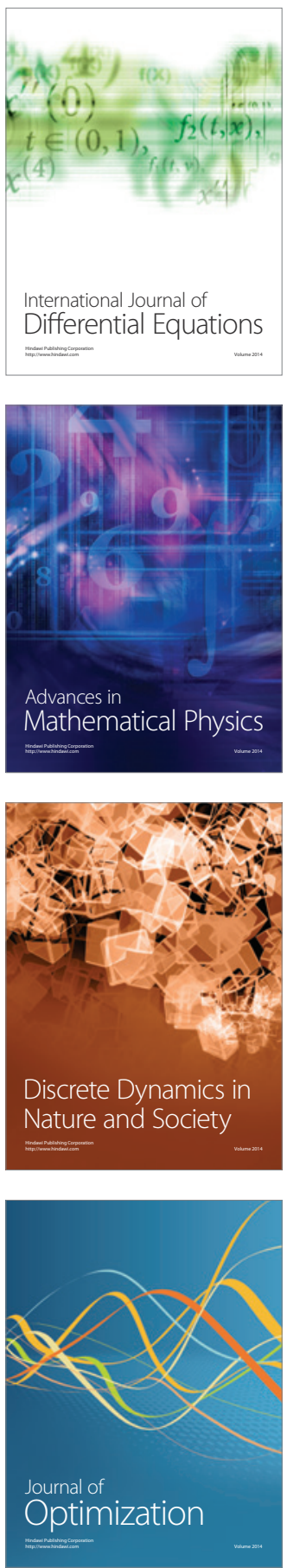